\title{
Look on the bright side
}

IEEE Trans. Comput. https://doi.org/10.1109/ TC.2018.2875986 (2018)

Dark silicon refers to the portion of a deeply scaled integrated circuit (IC) that cannot be operated at full capacity due to thermal constraints. Maximizing the allowed use of power (the power budget) across IC systems such as multi- or many-core systems will enable the performance of ICs with dark silicon to be maximized. However, determining an optimum power budget strategy is computationally intensive because of the complexity of the circuits involved. Existing approaches to approximate power budgets tend to be overly pessimistic, since they only consider steady state power and thermal relationships. Hai Wang and colleagues now propose a dynamic power budgeting method with low computational complexity called Greedy based Dynamic Power that accounts for current temperature states and transient thermal effects.

The researchers - who are from the University of Electronic Science and Technology of China in Chengdu, Cadence Design Systems Inc, and the University of California, Riverside - demonstrate that dynamic power budgeting leads to less pessimistic power budgeting. Moreover, in their theoretical and experimental investigations, Wang and colleagues demonstrate that dynamic power budgeting outperforms state-of-the-art power budgeting approaches for dark silicon multi-core systems. Optimistic power budgets should allow multi- and many-core dark silicon systems to operate with greater performance.

\section{Michael Lee}

Published online: 13 December 2018

https://doi.org/10.1038/s41928-018-0185-y 